PROCEEDINGS OF THE

AMERICAN MATHEMATICAL SOCIETY

Volume 129, Number 1, Pages 145-154

S 0002-9939(00)05731-2

Article electronically published on July 27, 2000

\title{
WEYL-HEISENBERG FRAMES FOR SUBSPACES OF $L^{2}(R)$
}

\author{
PETER G. CASAZZA AND OLE CHRISTENSEN
}

(Communicated by David R. Larson)

Abstract. A Weyl-Heisenberg frame

$$
\left\{E_{m b} T_{n a} g\right\}_{m, n \in Z}=\left\{e^{2 \pi i m b(\cdot)} g(\cdot-n a)\right\}_{m, n \in Z}
$$

for $L^{2}(R)$ allows every function $f \in L^{2}(R)$ to be written as an infinite linear combination of translated and modulated versions of the fixed function $g \in$ $L^{2}(R)$. In the present paper we find sufficient conditions for $\left\{E_{m b} T_{n a} g\right\}_{m, n \in Z}$ to be a frame for $\overline{\operatorname{span}}\left\{E_{m b} T_{n a} g\right\}_{m, n \in Z}$, which, in general, might just be a subspace of $L^{2}(R)$. Even our condition for $\left\{E_{m b} T_{n a} g\right\}_{m, n \in Z}$ to be a frame for $L^{2}(R)$ is significantly weaker than the previous known conditions. The results also shed new light on the classical results concerning frames for $L^{2}(R)$, showing for instance that the condition $G(x):=\sum_{n \in Z}|g(x-n a)|^{2}>A>0$ is not necessary for $\left\{E_{m b} T_{n a} g\right\}_{m, n \in Z}$ to be a frame for $\overline{\operatorname{span}}\left\{E_{m b} T_{n a} g\right\}_{m, n \in Z}$. Our work is inspired by a recent paper by Benedetto and Li, where the relationship between the zero-set of the function $G$ and frame properties of the set of functions $\{g(\cdot-n)\}_{n \in Z}$ is analyzed.

\section{PREliminaries AND NOTAtion}

Let $\mathcal{H}$ denote a separable Hilbert space with the inner product $\langle\cdot, \cdot\rangle$ linear in the first entry. Let $I$ denote a countable index set.

We say that $\left\{g_{i}\right\}_{i \in I} \subseteq \mathcal{H}$ is a frame (for $\mathcal{H}$ ) if there exist constants $A, B>0$ such that

$$
A\|f\|^{2} \leq \sum_{i \in I}\left|\left\langle f, g_{i}\right\rangle\right|^{2} \leq B\|f\|^{2}, \forall f \in \mathcal{H}
$$

In particular a frame for $\mathcal{H}$ is complete, i.e., $\overline{\operatorname{span}}\left\{g_{i}\right\}_{i \in I}=\mathcal{H}$. In case $\left\{g_{i}\right\}_{i \in I}$ is not complete, $\left\{g_{i}\right\}_{i \in I}$ can still be a frame for the subspace $\overline{\operatorname{span}}\left\{g_{i}\right\}_{i \in I}$; in that case we say that $\left\{g_{i}\right\}_{i \in I}$ is a frame sequence. The numbers $A, B$ that appear in the definition of a frame are called frame bounds.

Orthonormal bases and, more generally, Riesz bases are frames. Recall that $\left\{g_{i}\right\}_{i \in I}$ is a Riesz basis for $\mathcal{H}$ if $\overline{\operatorname{span}}\left\{g_{i}\right\}_{i \in I}=\mathcal{H}$ and

$$
\exists A, B>0: \quad A \sum_{i \in I}\left|c_{i}\right|^{2} \leq\left\|\sum_{i \in I} c_{i} g_{i}\right\|^{2} \leq B \sum_{i \in I}\left|c_{i}\right|^{2}, \quad \forall\left\{c_{i}\right\}_{i \in I} \in \ell^{2}(I) .
$$

If $\left\{g_{i}\right\}_{i \in I}$ is a Riesz basis for $\overline{\operatorname{span}}\left\{g_{i}\right\}_{i \in I}$, we say that $\left\{g_{i}\right\}_{i \in I}$ is a Riesz sequence.

Received by the editors March 10, 1999.

1991 Mathematics Subject Classification. Primary 42C15.

The first author was supported by NSF grant DMS 970618 and the second author by the Danish Research Council. The second author also thanks the University of Charlotte, NC, and the University of Missouri-Columbia, MO, for providing good working conditions. 
The present paper deals with frames having a special structure: all elements are translated and/or modulated versions of a single function. Let $L^{2}(R)$ denote the Hilbert space of functions on the real line which are square integrable with respect to the Lebesgue measure. First, define the following operators on functions $f \in L^{2}(R):$

$$
\begin{gathered}
\text { Translation by } a \in R: \quad\left(T_{a} f\right)(x)=f(x-a), x \in R . \\
\text { Modulation by } b \in R: \quad\left(E_{b} g\right)(x)=e^{2 \pi i b x} f(x), x \in R .
\end{gathered}
$$

A frame for $L^{2}(R)$ of the form $\left\{E_{m b} T_{n a} g\right\}_{m, n \in Z}$ is called a Weyl-Heisenberg frame (or Gabor frame). For a collection of different papers concerning those frames we refer to the monograph 5 .

Sufficient conditions for $\left\{E_{m b} T_{n a} g\right\}_{m, n \in Z}$ to be a frame for $L^{2}(R)$ has been known for about 10 years. The basic insight was provided by Daubechies [3. A slight improvement was proved in [6]:

Theorem 1.1. Let $g \in L^{2}(R)$ and suppose that

$$
\begin{aligned}
& \exists A, B>0: \quad A \leq \sum_{n \in Z}|g(x-n a)|^{2} \leq B \text { for a.e. } x \in R, \\
& \lim _{b \rightarrow 0} \sum_{k \neq 0}\left\|\sum_{n \in Z} T_{n a} g T_{n a+\frac{k}{b}} \bar{g}\right\|_{\infty}=0 .
\end{aligned}
$$

Then there exists $b_{0}>0$ such that $\left\{E_{m b} T_{n a} g\right\}_{m, n \in Z}$ is a Weyl-Heisenberg frame for $L^{2}(R)$ for all $\left.b \in\right] 0 ; b_{0}[$.

The proof of Theorem 1.1 is based on the following identity, valid for all continuous functions $f$ with compact support whenever $g$ satisfies (1):

$$
\begin{aligned}
& \sum_{m, n \in Z}\left|\left\langle f, E_{m b} T_{n a} g\right\rangle\right|^{2} \\
= & \frac{1}{b} \int|f(x)|^{2} G(x) d x \\
+ & \frac{1}{b} \sum_{k \neq 0} \int \overline{f(x)} f(x-k / b) \sum_{n \in Z} g(x-n a) \overline{g(x-n a-k / b)} d x .
\end{aligned}
$$

An estimate of the second term in (3) now shows that $\left\{E_{m b} T_{n a} g\right\}_{m, n \in Z}$ is actually a frame for all values of $b$ for which

$$
\sum_{k \neq 0}\left\|\sum_{n \in Z} T_{n a} g T_{n a+\frac{k}{b}} \bar{g}\right\|_{\infty}<A
$$

A more recent result can be found in [4]: in Theorem 2.3 it is proved that if (1) is satisfied and there exists a constant $D<A$ such that

$$
\sum_{k \neq 0} \sum_{n \in Z}\left|g(x-n a) g\left(x-n a-\frac{k}{b}\right)\right| \leq D \text { for a.e. } x \in R,
$$

then $\left\{E_{m b} T_{n a} g\right\}_{m, n \in Z}$ is a frame for $L^{2}(R)$ with bounds $\frac{A-D}{b}, \frac{B+D}{b}$. The reader should observe that [4] does not provide us with a generalization of the results in 3], 6] in a strict sense: there are cases where (5) is satisfied but (4) is not, and vice versa. The main point is that other conditions (that are easy to check) for $\left\{E_{m b} T_{n a} g\right\}_{m, n \in Z}$ to be a frame can be derived from (5); cf. Theorem 2.4 in [4]. 
Define the Fourier Transform $\mathcal{F}(f)=\hat{f}$ of $f \in L^{1}(R)$ by

$$
\hat{f}(y)=\int f(x) e^{-2 \pi i y x} d x .
$$

As usual we extend the Fourier Transform to an isometry from $L^{2}(R)$ onto $L^{2}(R)$. We denote the inverse Fourier transformation of $g \in L^{2}(R)$ by $\mathcal{F}^{-1} g$ or $\check{g}$. It is important to observe the following comutator relations, valid for all $a \in R$ :

$$
\mathcal{F} T_{a}=E_{-a} \mathcal{F}, \quad \mathcal{F} E_{a}=T_{a} \mathcal{F} .
$$

We need a result from [2]. The basic insight was provided by Benedetto and Li [1], who treated the case $a=1$.

Theorem 1.2. Let $g \in L^{2}(R)$. Then $\left\{T_{n a} g\right\}_{n \in Z}$ is a frame sequence with bounds $A, B$ if and only if

$$
0<a A \leq \sum_{n \in Z}\left|\hat{g}\left(\frac{x+n}{a}\right)\right|^{2} \leq a B \text { for a.e. } x \text { for which } \sum_{n \in Z}\left|\hat{g}\left(\frac{x+n}{a}\right)\right|^{2} \neq 0 .
$$

In that case $\left\{T_{n a} g\right\}_{n \in Z}$ is a Riesz sequence if and only if the set of $x$ for which $\sum_{n \in Z}\left|\hat{g}\left(\frac{x+n}{a}\right)\right|^{2}=0$ has measure zero.

Theorem 1.2 leads immediately to an equivalent condition to (1). Define the function $G$ and its kernel $N_{G}$ by

$$
\begin{aligned}
& G: R \rightarrow[0, \infty], \quad G(x):=\sum_{n \in Z}|g(x-n a)|^{2}, \\
& N_{G}=\{x \in R \mid G(x)=0\} .
\end{aligned}
$$

Corollary 1.3. $\left\{E_{\frac{n}{a}} g\right\}_{n \in Z}$ is a frame sequence with bounds $A, B$ if and only if

$$
0<\frac{A}{a} \leq \sum_{n \in Z}|g(x-n a)|^{2} \leq \frac{B}{a} \text { for a.e. } x \in R-N_{G} \text {. }
$$

In that case $\left\{E_{\frac{n}{a}} g\right\}_{n \in Z}$ is a Riesz sequence iff $N_{G}$ has measure zero.

Proof. The inequality

$$
0<\frac{A}{a} \leq \sum_{n \in Z}|g(x-n a)|^{2} \leq \frac{B}{a} \text { for a.e. } x \in R-N_{G}
$$

holds if and only if

$$
0<\frac{A}{a} \leq \sum_{n \in Z}|g([x-n] a)|^{2} \leq \frac{B}{a} \text { for a.e. } x \in R-N_{G} .
$$

By Theorem 1.2, (6) is equivalent to $\left\{T_{\frac{n}{a}} \breve{g}\right\}_{n \in Z}$ being a frame sequence with bounds $A, B$. Applying the Fourier transformation this is equivalent to $\left\{E_{\frac{n}{a}} g\right\}_{n \in Z}$ being a frame sequence with bounds $A, B$.

\section{The RESUlts}

From now on we concentrate on Weyl-Heisenberg frames $\left\{E_{m b} T_{n a} g\right\}_{m, n \in Z}$. Our first result gives a sufficient condition for $\left\{E_{m b} T_{n a} g\right\}_{m, n \in Z}$ to be a frame sequence. It can be considered as a "subspace version" of a result by Ron and Shen; cf. [7]. Our condition for $\left\{E_{m b} T_{n a} g\right\}_{m, n \in Z}$ to be a frame for $L^{2}(R)$ is significantly weaker than the conditions mentioned in section 1.

Let $L^{2}\left(R-N_{G}\right)$ denote the set of functions in $L^{2}(R)$ that vanishes at $N_{G}$. 
Theorem 2.1. Let $g \in L^{2}(R), a, b>0$ and suppose that

$$
\begin{aligned}
& \left.A:=\inf _{x \in[0, a]-N_{G}}\left[\sum_{n \in Z}|g(x-n a)|^{2}-\sum_{k \neq 0} \mid \sum_{n \in Z} g(x-n a) \overline{g\left(x-n a-\frac{k}{b}\right.}\right) \mid\right]>0 \\
& B:=\sup _{x \in[0, a]} \sum_{k \in Z}\left|\sum_{n \in Z} g(x-n a) \overline{g\left(x-n a-\frac{k}{b}\right)}\right|<\infty .
\end{aligned}
$$

Then $\left\{E_{m b} T_{n a} g\right\}_{m, n \in Z}$ is a frame for $L^{2}\left(R-N_{G}\right)$ with bounds $\frac{A}{b}, \frac{B}{b}$.

Proof. First, observe that $\overline{\operatorname{span}}\left\{E_{m b} T_{n a} g\right\}_{m, n \in Z} \subseteq L^{2}\left(R-N_{G}\right)$. Now consider a function $f \in L^{2}\left(R-N_{G}\right)$ which is bounded and has support in a compact set. The Heil-Walnut argument (3) is valid under the assumption (8) and it gives that

$$
\begin{aligned}
& \sum_{m, n \in Z}\left|\left\langle f, E_{m b} T_{n a} g\right\rangle\right|^{2} \\
= & \frac{1}{b} \int|f(x)|^{2} \sum_{n \in Z}|g(x-n a)|^{2} d x \\
+ & \frac{1}{b} \sum_{k \neq 0} \int \overline{f(x)} f(x-k / b) \sum_{n \in Z} g(x-n a) \overline{g(x-n a-k / b)} d x .
\end{aligned}
$$

We want to estimate the second term above. For $k \in Z$, define

$$
H_{k}(x):=\sum_{n \in Z} T_{n a} g(x) \overline{T_{n a+k / b} g(x)} .
$$

First, observe that

$$
\begin{aligned}
& \sum_{k \neq 0}\left|T_{-k / b} H_{k}(x)\right| \\
= & \sum_{k \neq 0}\left|T_{-k / b} \sum_{n \in Z} T_{n a} g(x) \overline{T_{n a+k / b} g(x)}\right| \\
= & \sum_{k \neq 0}\left|\sum_{n \in Z} T_{n a-k / b} g(x) \overline{T_{n a} g(x)}\right| \\
= & \sum_{k \neq 0}\left|\sum_{n \in Z} T_{n a+k / b} g(x) \overline{T_{n a} g(x)}\right| \\
= & \sum_{k \neq 0}\left|\sum_{n \in Z} \overline{T_{n a+k / b} g(x)} T_{n a} g(x)\right| \\
= & \sum_{k \neq 0}\left|H_{k}(x)\right| .
\end{aligned}
$$


Now, by a slight modification of the argument in [4, Theorem 2.3,

$$
\begin{aligned}
& \left|\sum_{k \neq 0} \int \overline{f(x)} f(x-k / b) \sum_{n \in Z} g(x-n a) \overline{g(x-n a-k / b)} d x\right| \\
\leq & \sum_{k \neq 0} \int|f(x)| \cdot\left|T_{k / b} f(x)\right| \cdot\left|H_{k}(x)\right| d x \\
= & \sum_{k \neq 0} \int|f(x)| \sqrt{\left|H_{k}(x)\right|} \cdot\left|T_{k / b} f(x)\right| \sqrt{\left|H_{k}(x)\right|} d x \\
\leq & \sum_{k \neq 0}\left(\int|f(x)|^{2}\left|H_{k}(x)\right| d x\right)^{1 / 2}\left(\int\left|T_{k / b} f(x)\right|^{2}\left|H_{k}(x)\right| d x\right)^{1 / 2} \\
\leq & \left(\sum_{k \neq 0} \int|f(x)|^{2}\left|H_{k}(x)\right| d x\right)^{1 / 2} \cdot\left(\sum_{k \neq 0} \int\left|T_{k / b} f(x)\right|^{2}\left|H_{k}(x)\right| d x\right)^{1 / 2} \\
= & \left(\int|f(x)|^{2} \sum_{k \neq 0}\left|H_{k}(x)\right| d x\right)^{1 / 2} \cdot\left(\int|f(x)|^{2} \sum_{k \neq 0}\left|T_{-k / b} H_{k}(x)\right| d x\right)^{1 / 2} \\
= & \int|f(x)|^{2} \sum_{k \neq 0}\left|H_{k}(x)\right| d x .
\end{aligned}
$$

Note that $\sum_{k \neq 0}\left|H_{k}(x)\right|=\sum_{k \neq 0}\left|\sum_{n \in Z} T_{n a} g(x) \overline{T_{n a+k / b} g(x)}\right|$ is a periodic function with period $a$. By (3) and the assumption (7) we now have

$$
\begin{aligned}
& \sum_{m, n \in Z}\left|\left\langle f, E_{m b} T_{n a} g\right\rangle\right|^{2} \\
\geq & \frac{1}{b} \int|f(x)|^{2}\left[\sum_{n \in Z}|g(x-n a)|^{2}-\sum_{k \neq 0} \mid \sum_{n \in Z} g(x-n a) \overline{g\left(x-n a-\frac{k}{b}\right)}\right] d x \\
\geq & \frac{A}{b}|| f \|^{2} .
\end{aligned}
$$

Similary, by (3) and (8),

$$
\begin{aligned}
& \sum_{m, n \in Z}\left|\left\langle f, E_{m b} T_{n a} g\right\rangle\right|^{2} \\
\leq & \frac{1}{b} \int|f(x)|^{2}\left[\sum_{n \in Z}|g(x-n a)|^{2}+\sum_{k \neq 0} \mid \sum_{n \in Z} g(x-n a) \overline{g\left(x-n a-\frac{k}{b}\right)}\right] d x \\
= & \frac{1}{b} \int|f(x)|^{2} \sum_{k \in Z} \mid \sum_{n \in Z} g(x-n a) \overline{g\left(x-n a-\frac{k}{b}\right)} \\
\leq & \frac{B}{b} \|\left. f\right|^{2} .
\end{aligned}
$$

Since those two estimates holds on a dense subset of $L^{2}\left(R-N_{G}\right)$, they hold on $L^{2}\left(R-N_{G}\right)$. Thus $\left\{E_{m b} T_{n a} g\right\}_{m, n \in Z}$ is a frame for $L^{2}\left(R-N_{G}\right)$ with the desired bounds. 
The advantage of Theorem 2.1 compared to the results in section 1 is that we compare the functions $\sum_{n \in Z}|g(x-n a)|^{2}$ and $\sum_{k \neq 0}\left|H_{k}(x)\right|$ pointwise rather than assuming that the supremum of $\sum_{k \neq 0}\left|H_{k}(x)\right|$ is smaller than the infimum of $\sum_{n \in Z}|g(x-n a)|^{2}$. It is easy to give concrete examples where Theorem 2.1 shows that $\left\{E_{m b} T_{n a} g\right\}_{m, n \in Z}$ is a frame for $L^{2}(R)$ but where the conditions in section 1 are not satisfied:

Example. Let $a=b=1$ and define

$$
g(x)= \begin{cases}1+x & \text { if } x \in[0,1[ \\ \frac{1}{2} x & \text { if } x \in[1,2[ \\ 0 & \text { otherwise. }\end{cases}
$$

For $x \in[0,1[$ we have

$$
G(x)=\sum_{n \in Z}|g(x-n)|^{2}=g(x)^{2}+g(x+1)^{2}=\frac{5}{4}(x+1)^{2}
$$

and

$$
\sum_{k \neq 0}\left|\sum_{n \in Z} g(x-n) \overline{g(x-n-k)}\right|=(1+x)^{2},
$$

so by Theorem $2.1\left\{E_{m} T_{n} g\right\}_{m, n \in Z}$ is a frame for $L^{2}(R)$ with bounds $A=\frac{1}{4}, B=9$. But $\inf _{x \in R} G(x)=\frac{5}{4}$ and

$$
\sum_{k \neq 0}\left\|\sum_{n \in Z} T_{n} g T_{n+k} \bar{g}\right\|_{\infty}=4
$$

so the condition (4) is not satisfied. (5) is not satisfied either.

Remark. It is well known that $G$ being bounded below is a necessary condition for $\left\{E_{m b} T_{n a} g\right\}_{m, n \in Z}$ to be a frame for $L^{2}(R)$; cf. [3]. Theorem 2.1 shows that this condition is not necessary for $\left\{E_{m b} T_{n a} g\right\}_{m, n \in Z}$ to be a frame sequence. However, it is implicit in (7) that $G$ has to be bounded below on $R-N_{G}$ in order for Theorem 2.1 to work, and an easy modification of the proof in [3] shows that this is actually a necessary condition for $\left\{E_{m b} T_{n a} g\right\}_{m, n \in Z}$ to be a frame for $L^{2}\left(R-N_{G}\right)$. We shall later give examples of frame sequences for which $G$ is not bounded below on $R-N_{G}$.

In case $g$ has support in an interval of length $\frac{1}{b}$ an equivalent condition for $\left\{E_{m b} T_{n a} g\right\}_{m, n \in Z}$ to be a frame sequence can be given. First, observe that by (3) this condition on $g$ implies that for all continuous functions $f$ with compact support, we have

$$
\sum_{m, n \in Z}\left|\left\langle f, E_{m b} T_{n a} g\right\rangle\right|^{2}=\frac{1}{b} \int|f(x)|^{2} G(x) d x .
$$

It is not hard to show that this actually holds for all $f \in L^{2}(R)$; cf. [6].

Corollary 2.2. Suppose that $g \in L^{2}(R)$ has compact support in an interval I of length $|I| \leq 1 / b$. Then $\left\{E_{m b} T_{n a} g\right\}_{m, n \in Z}$ is a frame sequence with bounds $A, B$ if and only if

$$
0<b A \leq \sum_{n \in Z}|g(x-n a)|^{2} \leq b B, \text { for a.e. } x \in R-N_{G} .
$$

In that case $\left\{E_{m b} T_{n a} g\right\}_{m, n \in Z}$ is actually a frame for $L^{2}\left(R-N_{G}\right)$. 
Proof. Suppose that $g$ has support in an interval $I$ of length $|I| \leq \frac{1}{b}$. If $0<b A \leq$ $G(x) \leq b B$ for a.e. $x \in R-N_{G}$, it follows from Theorem 2.1 that $\left\{E_{m b} T_{n a} g\right\}_{m, n \in Z}$ is a frame sequence with the desired bounds. Now suppose that $\left\{E_{m b} T_{n a} g\right\}_{m, n \in Z}$ is a frame sequence with bounds $A, B$. Then, for every interval $I$ of length $|I|=1 / b$ and every function $f \in L^{2}(I)$,

$$
\sum_{m, n}\left|\left\langle f, E_{m b} T_{n a} g\right\rangle\right|^{2}=\frac{1}{b} \int_{R}|f(x)|^{2} G(x) d x \leq B\|f\|^{2} .
$$

But this is clearly equivalent to

$$
G(x)=\sum_{n \in Z}|g(x-n a)|^{2} \leq B b \text { a.e. }
$$

To prove the lower bound for $G$ we proceed by way of contradiction. Suppose that for some $\epsilon>0$ we have $0<G(x) \leq(1-\epsilon) A b$ on a set of positive measure. In this case there is a set $\Delta$ of positive measure and supported in an interval of length $\leq \frac{1}{b}$ so that $0<G(x) \leq(1-\epsilon) A b$ on $\Delta$. Then, for any function $f \in L^{2}(R)$ supported on $\Delta$, we have

$$
\begin{aligned}
& \sum_{m, n}\left|\left\langle f, E_{m b} T_{n a} g\right\rangle\right|^{2}=\frac{1}{b} \int_{R}|f(x)|^{2} G(x) d x \\
& \leq \frac{(1-\epsilon) A b}{b} \int_{R}|f(x)|^{2} d x=(1-\epsilon) A\|f\|^{2} .
\end{aligned}
$$

Since $G(x)>0$ on $\Delta$, there is a $k \in Z$ so that $\chi_{\Delta} T_{k a} g$ is not the zero function. With $\Delta^{\prime}:=\Delta \cap \operatorname{Supp}\left(T_{k a} g\right)$ we have

$$
f:=\chi_{\Delta^{\prime}} T_{k a} g \in \overline{\operatorname{span}}\left\{E_{m b} T_{k a} g\right\}_{m \in Z} \subseteq \overline{\operatorname{span}}\left\{E_{m b} T_{n a} g\right\}_{m, n \in Z},
$$

so the above calculation shows that the lower bound for $\left\{E_{m b} T_{n a} g\right\}_{m, n \in Z}$ is at most $(1-\epsilon) A$, which is a contradiction. Thus

$$
G(x) \geq b A \text { for a.e. } x \in R-N_{G} \text {. }
$$

In case the condition in Corollary 2.2 is satisfied, it follows from Theorem 2.1 that $\left\{E_{m b} T_{n a} g\right\}_{m, n \in Z}$ is a frame for $L^{2}\left(R-N_{G}\right)$.

For functions $g$ with the property that the translates $T_{n a} g, n \in Z$, have disjoint support we can give an equivalent condition for $\left\{E_{m b} T_{n a} g\right\}_{m, n \in Z}$ to be a frame sequence. Define the function

$$
\tilde{G}(x): R \rightarrow[0, \infty], \quad \tilde{G}(x)=\sum_{m \in Z}\left|g\left(x+\frac{m}{b}\right)\right|^{2} .
$$

Proposition 2.3. Let $g \in L^{2}(R), a, b>0$ and suppose that

$$
\operatorname{supp}(g) \cap \operatorname{supp}\left(T_{n a} g\right)=\emptyset, \forall n \in Z-\{0\} .
$$

Then $\left\{E_{m b} T_{n a} g\right\}_{m, n \in Z}$ is a frame sequence with bounds $A, B$ if and only if there exist $A, B>0$ such that

$$
b A \leq \sum_{m \in Z}\left|g\left(x+\frac{m}{b}\right)\right|^{2} \leq b B \text { for a.e. } x \in R-N_{\tilde{G}} .
$$

In that case, $\left\{E_{m b} T_{n a} g\right\}_{m, n \in Z}$ is a Riesz sequence iff $N_{\tilde{G}}$ has measure zero. 
Proof. Because of the support condition (9), it is clear that $\left\{E_{m b} g\right\}_{m \in Z}$ is a frame sequence iff $\left\{E_{m b} T_{n a} g\right\}_{m, n \in Z}$ is a frame sequence, in which case the sequences have the same frame bounds. But by Corollary $1.3\left\{E_{m b} g\right\}_{m \in Z}$ is a frame sequence with bounds $A, B$ iff

$$
b A \leq \sum_{m \in Z}\left|g\left(x+\frac{m}{b}\right)\right|^{2} \leq b B \text { for a.e. } x \in R-N_{\tilde{G}} .
$$

Also, $\left\{E_{m b} T_{n a} g\right\}_{m, n \in Z}$ is a Riesz sequence iff $\left\{E_{m b} g\right\}_{m \in Z}$ is a Riesz sequence, which, by Corollary 1.3, is the case iff $N_{\tilde{G}}$ has measure zero.

We are now ready to show that $G$ being bounded below on $R-N_{G}$ (by a positive number) is not a necessary condition for $\left\{E_{m b} T_{n a} g\right\}_{m, n \in Z}$ to be a frame sequence.

Example. Let $a, b>0$ and suppose that $\frac{1}{a b} \notin N$. Chose $\epsilon>0$ such that

$$
[0, \epsilon]+n a \cap\left[\frac{1}{b}, \frac{1}{b}+\epsilon\right]=\emptyset, \forall n \in Z .
$$

This implies that $\epsilon<\min \left(a, \frac{1}{b}\right)$. Define

$$
g(x):= \begin{cases}x & \text { if } x \in[0, \epsilon], \\ \sqrt{1-\left(x-\frac{1}{b}\right)^{2}} & \text { if } x \in\left[\frac{1}{b}, \frac{1}{b}+\epsilon\right], \\ 0 & \text { otherwise. }\end{cases}
$$

Then the condition (9) in Proposition 2.3 is satisfied. Also, for $x \in[0, \epsilon]$,

$$
\tilde{G}(x)=\sum_{m \in Z}\left|g\left(x+\frac{m}{b}\right)\right|^{2}=g(x)^{2}+g(x+1)^{2}=1
$$

and for $\left.x \in] \epsilon, \frac{1}{b}\right]$, we have $\tilde{G}(x)=0$. Thus, by Proposition $2.3\left\{E_{m b} T_{n a} g\right\}_{m, n \in Z}$ is a frame sequence. But for $x \in[0, \epsilon]$,

$$
G(x)=\sum_{n \in Z}|g(x-n a)|^{2}=x^{2} .
$$

Thus $G$ is not bounded below by a positive number on $R-N_{G}$. By the remark after Theorem 2.1 this implies that $\overline{\operatorname{span}}\left\{E_{m b} T_{n a} g\right\}_{m, n \in Z} \neq L^{2}\left(R-N_{G}\right)$.

For $a b>1$ it is even possible to construct an orthonormal sequence having all the features of the above example. For example, let $a=2, b=1$ and

$$
g(x):= \begin{cases}x & \text { if } x \in[0,1] \\ \sqrt{2 x-x^{2}} & \text { if } x \in] 1,2] \\ 0 & \text { otherwise }\end{cases}
$$

Since

$$
\sum_{m \in Z}\left|g\left(x+\frac{m}{b}\right)\right|^{2}=1, \forall x
$$

it follows by Proposition 2.3 that $\left\{E_{m} T_{2 n} g\right\}_{m, n \in Z}$ is a Riesz sequence with bounds $A=B=1$, which implies that $\left\{E_{m} T_{2 n} g\right\}_{m, n \in Z}$ is an orthonormal sequence. But $G(x)=\sum_{n \in Z}|g(x-2 n)|^{2}$ is not bounded below on $R-N_{G}$.

$G$ being bounded above is still a necessary condition for $\left\{E_{m b} T_{n a} g\right\}_{m, n \in Z}$ to be a frame sequence (repeat the argument in Corollary 2.2). $\tilde{G}$ also has to be bounded above: 
Proposition 2.4. If $\left\{E_{m b} T_{n a} g\right\}_{m, n \in Z}$ is a frame sequence with upper bound $B$, then

$$
\sum_{m \in Z}\left|g\left(x+\frac{m}{b}\right)\right|^{2} \leq B \quad \text { a.e. }
$$

Proof. If $\left\{E_{m b} T_{n a} g\right\}_{m, n \in Z}$ is a frame sequence, then $\left\{\mathcal{F}^{-1} E_{m b} T_{n a} g\right\}_{m, n \in Z}=$ $\left\{T_{m b} E_{n a} \breve{g}\right\}_{m, n \in Z}$ is a frame sequence with the same bounds. In particular the sequence $\left\{T_{m b} \check{g}\right\}_{m, n \in Z}$ has the upper frame bound $B$. By Theorem 1.2 (or, more precisely, the proof of it in 2]) it follows that

$$
\sum_{m \in Z}\left|g\left(\frac{x+m}{b}\right)\right|^{2} \leq B \text { for a.e. } x .
$$

It follows that $\sum_{m \in Z}\left|g\left(x+\frac{m}{b}\right)\right|^{2} \leq B$ a.e.

Remark. Recall that a wavelet frame for $L^{2}(R)$ has the form

$$
\left\{\frac{1}{a^{n / 2}} g\left(\frac{x}{a^{n}}-m b\right)\right\}_{m, n \in Z},
$$

where $a>1, b>0$ and $g \in L^{2}(R)$ are fixed.

As well as Weyl-Heisenberg frames, wavelet frames play a very important role in applications. The theory for the two types of frames was developed at the same time, with the main contribution due to Daubechies. Several results for WeylHeisenberg frames have counterparts for wavelet frames. For example, Theorem 5.1.6 in [6] gives sufficient conditions for $\left\{\frac{1}{a^{n / 2}} g\left(\frac{x}{a^{n}}-m b\right)\right\}_{m, n \in Z}$ to be a frame based on a calculation similar to (3).

Also our results for Weyl-Heisenberg frames have counterparts for wavelet frames. The ideas in the proof of Theorem 2.1 can be used to modify [6], Theorem 5.1.6, which leads to the following:

Theorem 2.5. Let $a>1, b>0$ and $g \in L^{2}(R)$ be given. Let

$$
N:=\left\{\left.\gamma \in[1, a]\left|\sum_{n \in Z}\right| \hat{g}\left(a^{n} \gamma\right)\right|^{2}=0\right\}
$$

and suppose that

$$
\begin{aligned}
& A:=\inf _{|\gamma| \in[1, a]-N}\left[\sum_{n \in Z}\left|\hat{g}\left(a^{n} \gamma\right)\right|^{2}-\sum_{k \neq 0} \sum_{n \in Z}\left|\hat{g}\left(a^{n} \gamma\right) \hat{g}\left(a^{n} \gamma+k / b\right)\right|\right]>0, \\
& B:=\sup _{|\gamma| \in[0, a]} \sum_{k, n \in Z}\left|\hat{g}\left(a^{n} \gamma\right) \hat{g}\left(a^{n} \gamma+k / b\right)\right|<\infty .
\end{aligned}
$$

Then $\left\{\frac{1}{a^{n / 2}} g\left(\frac{x}{a^{n}}-m b\right)\right\}_{m, n \in Z}$ is a frame sequence with bounds $\frac{A}{b}, \frac{B}{b}$.

\section{ACKNOWLEDGMENT}

The authors would like to thank Christopher Heil for discussions and Baiqiao Deng for giving us access to the preprint [4]. 


\section{REFERENCES}

[1] Benedetto, J. and Li, S.: The theory of multiresolution analysis frames and applications to filter banks. Appl. Comp. Harm. Anal. 5 (1998), 389-427. MR 99k:42054

[2] Casazza, P.G., Christensen, O. and Kalton, N.: Frames of translates, Collectanea Mathematica, accepted for publication.

[3] Daubechies, I.: The wavelet transformation, time-frequency localization and signal analysis. IEEE Trans. Inform. Theory 36 (1990), p. 961-1005.

[4] Deng, B., Schempp, W., Xiao, C. and Wu, Z.: On the existence of Weyl-Heisenberg and affine frames. Preprint, 1997.

[5] Feichtinger, H.G. and Strohmer, T., (Eds.): Gabor analysis and algorithms: Theory and applications. Birkhäuser, 1998. MR 98h:42001

[6] Heil, C. and Walnut, D.: Continuous and discrete wavelet transforms. SIAM Review 31 (1989), p .628-666. MR 91c:42032

[7] Ron, A. and Shen, Z.: Weyl-Heisenberg systems and Riesz bases in $L^{2}(\mathbb{R})$, Duke Math. J. 89 (1997), 237-282. MR 98i:42013

[8] Young, R.: An introduction to nonharmonic Fourier series. Academic Press, New York, 1980. MR 81m:42027

Department of Mathematics, University of Missouri, Columbia, Missouri 65211

E-mail address: pete@casazza.math.missouri.edu

Department of Mathematics, Technical University of Denmark, Building 303, 2800 LyNGBY, DENMARK

E-mail address: ole.christensen@mat.dtu.dk 